\title{
Natural language as a window into the subjective effects and neurochemistry of psychedelic drugs
}

\author{
Enzo Tagliazucchi ${ }^{1,2, *}$
}

${ }^{1}$ Latin American Brain Health Institute (BrainLat), Universidad Adolfo Ibanez, Santiago, Chile

${ }^{2}$ Departamento de Física, Universidad de Buenos Aires and Instituto de Física de Buenos Aires (IFIBA - CONICET), Pabellón I, Ciudad Universitaria (1428), CABA, Buenos Aires, Argentina

*Corresponding author: enzo@ df.uba.ar, (+54 11) 5285-7530/7565/7566

\begin{abstract}
Psychedelics are drugs capable of eliciting profound alterations in the subjective experience of the users, sometimes with long-lasting consequences. Because of this, psychedelic research tends to focus on human subjects, given their capacity to construct detailed narratives about the contents of their consciousness experiences. In spite of its relevance, the interaction between serotonergic psychedelics and language production is comparatively understudied in the recent literature. This review is focused on two aspects of this interaction: how the acute effects of psychedelic drugs impact on speech organization regardless of its semantic content, and how to characterize the subjective effects of psychedelic drugs by analyzing the semantic content of written retrospective reports. We show that the computational characterization of language production is an emergent powerful tool to predict the therapeutic outcome of individual experiences, relate the effects elicited by psychedelics with those associated with other altered states of consciousness, draw comparisons between the psychedelic state and the symptomatology of certain psychiatric disorders, and investigate the neurochemical profile and mechanism of action of different psychedelic drugs. We conclude that researchers studying psychedelics can considerably expand the range of their potential scientific conclusions by analyzing brief interviews obtained before, during and after the acute effects. Finally, we list a series of questions and open problems that should be addressed to further consolidate this approach.
\end{abstract}

Keywords: psychedelics, language, consciousness, cognition, pharmacology, semantics 


\section{Introduction}

Both humans and other animals display a natural tendency to consume drugs that transiently modify their behavior, cognition and overall state of consciousness (Samorini, 2002). From an evolutionary standpoint, the reasons behind this inclination are not yet fully understood. What is certain is that, throughout history, some humans have adopted an exploratory attitude towards drugs, experiencing their effects and then communicating to others the nature of their subjective experiences by means of written or spoken language (Escohotado, 1999). The urge to report drug-induced experiences is especially notable for the serotonergic psychedelics, a class of compounds capable of eliciting deep modifications in the contents of consciousness, including changes to visual, auditory and somatosensory perception, distortions in the sense of self and in the spatial location and extent of body boundaries, increased emotional volatility, and mystical-type experiences, which encompass feelings of bliss, transcendence, ineffability and selflessness, among other defining characteristics (Nichols, 2016; Johnson et al., 2019; Preller and Vollenweider, 2016). Perhaps the most wellknown recollection of a drug-induced experience in Western literature, "The Doors of Perception" by Aldous Huxley, stems from the author's experimentation with mescaline, a naturally occurring psychedelic phenethylamine (Huxley, 1954).

Due to the complexity of the induced effects, it is almost impossible for users of psychedelic drugs to communicate the nature of their subjective experience without resorting to language. This is not necessarily true for other families of psychoactive drugs: the effects of sedatives or stimulants, for example, can be directly appreciated by the observation of behavior, which validates the use of animal models for their investigation (Gonçalves et al., 2021). In contrast, the subjective effects of psychedelics are not linked to rigid and characteristic behavioral patterns, since they can include states of excitation or relaxation depending on the background and intention of the user ("set"), as well as the surrounding environment ("setting") (Carhart-Harris et al., 2018). In other words, there is nothing in the behavior of an individual under the acute effects of $75 \mu \mathrm{g}$ of lysergic acid diethylamide (LSD, a potent psychedelic drug) that unequivocally indicates the presence of visual distortions or feelings of body boundlessness - only by explicit linguistic reports is this individual capable of communicating the full extent of the drug-induced subjective effects. While some recurrent behaviors are considered markers of psychedelic drug action in animal models (such as 
the head twitch) (Hanks and González-Maeso, 2013) the general consensus is that human research is necessary to achieve a complete understanding of the neuropharmacology of serotonergic psychedelics (Nichols, 2016).

Clearly, language plays a central role in the investigation of serotonergic psychedelics, yet studies of the interaction between these drugs and language production are relatively underrepresented in the literature. Our goal is to review studies supporting the analysis of natural language as an important window into the subjective effects elicited by psychedelic drugs, their neurochemical mechanisms of action, and their effects on the large-scale brain networks supporting human cognitive function and consciousness. To introduce the need of studying verbal or written reports of subjective experiences, we will first discuss some of the unique methodological problems raised by psychedelic drugs, and briefly mention some of the advantages and limitations of unconstrained natural language reports compared to other tools to quantify subjective experience. Before conducting an exhaustive review of the literature, we will briefly introduce fundamentals of natural language processing (NLP), a set of computational tools used to extract meaningful quantitative information from reports produced by humans. Afterwards, we will review studies focusing on the semantic analysis of retrospective reports and the investigation of speech produced under the acute drug effects. After presenting an overview of how these different dimensions of analysis inform important aspects of the psychedelic experience, ranging from phenomenology to their underlying neurochemistry and neurophysiology, we will discuss a series of open problems that outline possible new directions for the use of natural language in the study of psychedelics.

\section{How should psychedelic drugs be studied?}

The consensus view is that psychedelic research must necessarily include human participants, yet what is the adequate methodology to investigate these participants and their subjective experiences? The left panel of Figure 1 contains a simple taxonomy of possible experimental approaches towards the study of these compounds. The first dimension (x axis) indexes the freedom that the participants have during the experiment, while the second (y axis) determines whether the experiment is oriented to measure their subjective experience, or to assess their performance in goal-oriented tasks. Ever since the earliest days of psychedelic research, it is clear that certain cognitive functions (e.g. attention) can be severely impaired during the effects of these drugs 
(Bayne and Carter, 2018), thus limiting the use of the standard approach of cognitive neuroscience (right lower quadrant in Figure 1, left), which is based on measuring the performance in tasks designed to evaluate specific domains of human cognition and their relationship with brain function. While some studies adopting this approach exist in the literature (Carter et al., 2005; Bouso et al., 2013; Pokorny et al., 2020; Healy, 2021), they are underrepresented in comparison to those seeking to determine the neural correlates of self-reported subjective effects. Impaired attention also limits the use of paradigms from psychophysics, as well as other approaches where the main outcome of the experiment is a metric of behavioral performance in an attentiondemanding task. Perhaps the most widely adopted approach for the study of psychedelic compounds in humans is that of self-reported questionnaires (right upper quadrant in Figure 1, left), including versions that can be completed even when undergoing intense effects, such as visual analogue scales (VAS). Over the past decade, an ample variety of questionnaires has been proposed to investigate how psychedelics affect cognition, conscious perception, thought processes, as well as beliefs, attitudes, and personality traits, and their results have been correlated with objective measurements of brain activity obtained using neuroimaging techniques such as functional magnetic resonance imaging (fMRI), electroencephalography (EEG) and magnetoencephalography (MEG) (Dittrich, 1998; Studerus et al., 2010; Barrett et al., 2015; Carhart-Harris et al., 2012; Muthukumaraswamy et al., 2013; Kometer et al., 2015; Carhart-Harris et al., 2016; Tagliazucchi et al., 2016; Barrett, et al., 2016; Nour et al., 2016; Preller et al., 2018; Kettner et al., 2019; Roseman et al., 2019; Timmermann et al., 2019; Pallavicini et al., 2020; Tagliazucchi et al., 2021) 

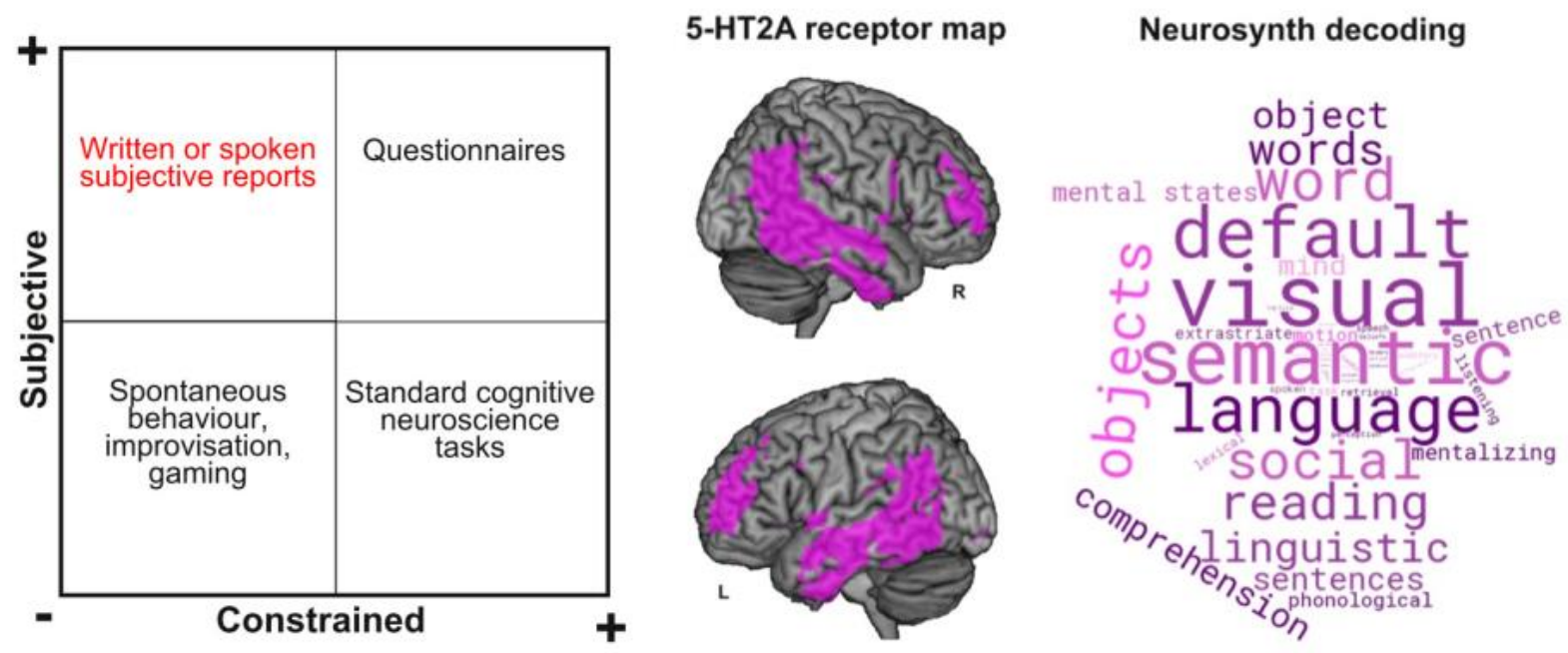

Figure 1. Natural language processing as a method to investigate the effects of psychedelic drugs. Left panel: Different ways of approaching the study of the effects of psychedelic drugs. The first dimension (x axis) indexes whether the experimental paradigm constrains subjects to adopt a set of predefined behavioral patterns (for instance, when interacting with the interface of a rigidly scripted cognitive experiment). The second dimension (y axis) indexes whether the experiment intends to gather information related to the subjective experience of the participants, or to measure their capacity to perform certain tasks or to achieve specific goals. Natural language reports, the main topic of this review, constitute a comparatively unconstrained method to investigate the effects of psychedelic drugs on subjective experience and spontaneous cognitive processing. Right panel: Overlap between regions presenting a high density of 5HT2A receptors and those consistently involved in language understanding and production, as determined by Neurosynth meta-analytic activation maps (Yarkoni et al., 2011). The 5-HT2A receptor density map (left) was obtained from 95 healthy subjects using Positron Emission Tomography (PET) with $\left[{ }^{18} \mathrm{~F}\right]$ altanserin as a radioligand (Saulin et al., 2012). The word cloud on the right was obtained using Neurosynth's decoder tool applied to the 5-HT2A receptor density map (https://neurosynth.org/decode/). Only terms associated with human cognitive functions were retained in the word cloud; those of neuroanatomical origin (e.g. cingulate cortex, insula) were discarded.

In comparison to paradigms based on tasks and self-reported measures, the study of natural and spontaneous goal-oriented behavior (left upper quadrant in Figure 1, left) is uncommon, mainly due to the difficulty of extracting meaningful objective and quantitative data from unstructured and unconstrained observations. When assessing the merits of verbal or written reports of subjective experiences, this limitation should also be considered a potential advantage, since the range of 
information that can be gathered by a questionnaire is limited by the choice of questions, while the completeness of subjective reports communicated via natural language is, in principle, only limited by the participants' capacities for recall and introspection. To further ensure the completeness of reports provided in natural language, guided interviews could help individuals to narrate their memories of the experience in an orderly and structured fashion, with techniques such as the microphenomenological interview presenting potential to accurately and reliably capture subjective experience with a level of detail beyond the scope of questionnaires or ordinary natural language reports (Petitmengin et al., 2019). Another advantage of unconstrained reports is their ubiquity. After Aldous Huxley's famous mescaline "trip report" in "The Doors of Perception" (Huxley, 1954), the practice of retrospectively narrating psychedelic experience steadily increased in popularity, eventually leading to large online repositories of such reports. A prominent example is found in Erowid's Experience Vaults (https://erowid.org/experiences/), which contain thousands of curated reports of drug-induced experiences across a wide range of chemicals and their combinations. While the usefulness of this data is hindered by several unknowns and limitations (which will be discussed later in this review), it nevertheless represents one of the largest sources of information concerning the acute effects of psychedelic drugs. Efforts are underway to systematize the results of psychometric questionnaires available in the scientific literature (such as the Altered States Database, http://www.asdb.info/; Schmidt and Berkemeyer, 2018), yet the amount of processed data is still small relative to the number of reports contained in the Erowid database.

Natural language reports obtained during the acute effects of psychedelics open a new dimension of analysis beyond the possibilities of psychometric questionnaires, given by the effects of the drugs on language itself (as opposed to their effects on the semantic content of the reports). As shown in the right panel of Figure 1, the distribution of regions with high density of 5-HT2A receptors (the main pharmacological target of psychedelics; Nichols, 2016) presents substantial overlap with brain activations elicited in neuroimaging experiments of language production and processing. The word cloud on the right of Figure 1 contains terms whose associated meta-analytic activation maps (obtained using Neurosynth, https://neurosynth.org/; Yarkoni et al., 2011) match the 5-HT2A receptor density map; notably, these terms include "semantic", "language", "words", and "reading", among others related to language. This suggests that 5-HT2A receptor activation 
can exert a direct modulatory function on the production and understanding of language. In this case, a psychedelic drug could modify certain features of language produced by an individual under its acute effects, including both semantic and non-semantic features. The content of subjective reports constitutes the message, while features related to the general structure of spoken language (regardless of the semantics) pertain to the medium, which is difficult to investigate solely using questionnaires. As we will discuss in this review, increasing evidence supports that certain language alterations are characteristic of specific neuropsychiatric disorders, which in turn could arise from neurochemical alterations at the level of networks of neurons. We will then argue that relating this body of research with the speech abnormalities observed under the effects of psychedelics could be informative to clarify their mechanism of action, as well as to inform some of their potential therapeutic uses.

\section{Fundamentals of natural language processing}

For natural language reports to be useful in the investigation of the phenomenology of psychedelic drugs, a necessary first step is to extract from them reliable and quantitative information. Since these reports are unstructured and unconstrained, their analysis is more challenging than that of questionnaires, for which standardized numerical outcomes are readily available. The methods used to extract information from this kind of data are collectively known as NLP (Chowdhury, 2003). We distinguish between the analysis of transcripts, focusing only on the text associated with a report, and the analysis of the acoustic features of spoken language. In this review, we will discuss only studies based on NLP applied to transcripts, since the acoustic analysis of drug-induced alterations to speech is almost unexplored in the literature. Nevertheless, we note that this direction of research is promising and should be explored in the future.

The analysis of written reports (or speech transcripts) can be further subdivided into two domains. Semantic analyses are concerned with inferring the meaning intended by the subjects, while nonsemantic analyses refer to other aspects of language that are, in general, independent of meaning; for instance, the relative frequency of different parts of speech (noun, pronoun, verb, adjective, adverb, preposition, conjunction, interjection), or the repetition of words or sequences of words, independently of the content they convey. 
In the context of this review, the interpretation of meaning is based on the distributional hypothesis of semantics (Sahlgren, 2006), stating that the similarity between the meaning of two words depends on their statistical co-occurrence in similar linguistic contexts (as expressed by Wittgenstein, “...the meaning of a word is its use in the language”) (Wittgenstein, 1953). Following this idea, two documents share semantic content if they contain similar words that also appear with similar frequencies. Defined in this way, the semantic similarity depends on the linguistic context used to estimate word co-occurrence; for instance, we expect that for New Yorkers, the semantic similarity between nouns "yellow" and "taxi" is higher than for Londoners, for whom taxis are frequently black instead of yellow.

Commonly, texts are first pre-processed to transform words to lemmas (i.e. lemmatization) to reduce the proliferation of different but semantically related terms, such as the different tenses of the same verb. Other common preprocessing steps include removal of rare or very frequent terms, including stopwords ("a", "the", "is", "are", etc.). Semantic analyses usually start from a representation of the data given by a term-document matrix, where rows correspond to the terms retained after preprocessing, and documents represent a collection of drug-induced subjective reports. The information about the terms and their use is included in this matrix, which contains in its $i, j$ entry the (normalized) count of the $\mathrm{i}$-th word in the $\mathrm{j}$-th document ${ }^{1}$. In principle, computing the similarity (e.g. cosine similarity, linear correlation, etc.) between the rows or columns of this matrix results in a measure of semantic similarity between the terms or documents, respectively. In practice, however, this direct approach is replaced by more sophisticated NLP methods capable of sorting two important obstacles (Landauer et al., 1998). First, if the vocabulary of terms is large, the entries of the matrix will be sparse, i.e. most of the entries will be zero. Similarity measures computed between rows and columns of a very sparse term-document matrix are likely to yield erroneous or misleading results. Second, the direct analysis of the term-document matrix results in semantic similarity estimates based on term co-occurrence between documents; however, two terms might never co-occur in the documents, but at the same time frequently occur together with a third term, thus being semantically related. For example, the number of words shared between the sentences "the garden was full of flowering roses" and "a vase with daisies sits on the table" is

\footnotetext{
${ }^{1}$ This can be extended to represent pairs of adjacent words in the matrix (bigrams), or more generally, sequences of $\mathrm{n}$ consecutive words ( $\mathrm{n}$-grams). However, increasingly larger text corpora are required to adequately sample n-grams as $\mathrm{n}$ increases.
} 
zero; however, both sentences are clearly related to the concept of "flowers", even if that word does not directly appear in them.

Latent semantic analysis (LSA) is a method that simultaneously solves these two problems by reducing the number of linearly independent rows in the term-document matrix, i.e. by lowering its rank (Landauer et al., 1998). This results in a less sparse representation where terms can have non-zero similarity even if they never co-occur through the documents (the same in the case of two documents whose words do not overlap). Afterwards, the similarity between pairs of documents can be estimated by computing the cosine distance or the correlation coefficient between the corresponding columns of the term-document matrix. LSA is based on a matrix algebra procedure known as singular value decomposition (SVD; Klema and Laub, 1980), a generalization of the eigenvalue decomposition used to factor a rectangular matrix as $\mathrm{A}=\mathrm{USV}$, where $\mathrm{S}$ is a square diagonal matrix with diagonal entries known as singular values. The number of non-zero singular values is the rank of matrix A. To reduce the rank of A to rank $\mathrm{k}$, the singular value decomposition can be replaced by $A_{k}=U_{k} S_{k} V_{k}$, with $A_{k}$ the reduced rank matrix, $S_{k}$ the square submatrix obtained by truncating matrix $S$ to keep only the k largest singular values, and matrices $U_{k}$ and $V_{k}$ keeping only the first k columns and rows of matrices $U$ and $V$, respectively.

The semantic similarity between words can also be estimated using a family of methods known as word embeddings. These methods can be used to map the vocabulary into a vector space of reduced dimensionality, with proximity within the vector space indicating semantic similarity and viceversa. Methods such as word2vec (Mikolov et al., 2013) are capable of learning this vector space from data using a shallow neural network trained to infer the words surrounding each term in the training vocabulary. Both LSA and word embeddings are behind some of the most promising NLPbased markers of altered thought processes in psychiatric patients; for instance, they can be used to define metrics of semantic coherence, i.e. the extent to which the semantic content of speech changes unexpectedly, instead of following a more continuous and predictable trajectory (Elvevåg et al., 2007; Bedi et al., 2015; Iter et al., 2018; Corcoran et al., 2018; Marggraf et al., 2018; Spencer et al., 2021). 
In contrast, non-semantic methods treat written texts or speech transcripts as sequences of tokens arranged according to syntactical rules, regardless of their meaning. Along this line, the method of speech graphs transforms texts into graphs or networks, where nodes correspond to words (before or after lemmatization) and the directed links indicate that one word follows another in the text. The topological properties of the resulting graphs contain information useful to characterize druginduced language alterations, as well as abnormalities specific to certain neuropsychiatric disorders (Mota et al., 2012; Mota et al., 2014; Bertola et al., 2014; Mota et al., 2017; Coelho et al., 2021). Some examples of these topological properties include the number of cycles of different length, the average shortest path in the graph, the maximum distance across all pairs of nodes (diameter), among others (Bullmore and Sporns, 2009).

As a general observation, semantic methods are useful to investigate retrospective subjective reports in terms of their content (for instance, what happened during the experience, how it felt to the subject, etc.) and to compare the experiences narrated in different reports in terms of their shared content. Non-semantic methods applied to these reports are unlikely to provide information beyond the individual traits or writing styles of the participants. However, non-semantic analyses are useful to determine how the overall structure of verbal expression is modified during the acute effects (the medium), regardless of the particular content that is being expressed (the message), which could inform the pharmacological modulation of language production networks in the brain.

\section{Language production under the acute effects of psychedelics}

Among their many potential uses and denominations, serotonergic psychedelics have been called "psychotomimetics" (meaning "psychosis-mimicking”) and investigated as agents capable of eliciting states of altered consciousness and cognition that are similar to psychosis (Nichols and Walter, 2020). Over the last decades, the computational analysis of speech production has shown great promise to detect and predict psychotic episodes; in particular, many these studies have found that the discourse of psychotic patients tends to show reduced semantic coherence in comparison to that produced by healthy individuals (Elvevåg et al., 2007; Bedi et al., 2015; Iter et al., 2018; Corcoran et al., 2018; Marggraf et al., 2018; Spencer et al., 2021). Consistent with their purported role as psychotomimetics, early studies demonstrated that psychedelics also render speech less predictable and enhance free-association (Amarel and Cheek, 1965), while a more recent study 
based on a picture naming task showed that LSD enhances semantic associativity (Family et al., 2016). Another recent work used psychometric questionnaires and language processing tasks to show that LSD affects the stream of thought at multiple levels, facets, and time points after infusion (Wießner et al., 2021). However, the characteristics of unconstrained speech produced during the acute effects of psychedelics remain relatively underexplored from the perspective of NLP, an approach that is becoming increasingly commonplace in computational psychiatry.

An exception can be found in recent work by Sanz and colleagues, where authors applied semantic and non-semantic methods to interviews conducted at two different time points after intravenous infusion of $75 \mu \mathrm{g}$ of LSD (Sanz et al., 2021). Semantic coherence (computed as the variance of the distance between the word2vec embeddings of pairs of subsequent words) was reduced during the acute effects of the drug, while speech graphs presented a more recurrent structure, with increased verbosity and decreased lexicon. Example speech graphs for LSD and placebo are shown in Figure 2 (top panel), where the scrambling effects of LSD on the organization of natural language can be directly appreciated. Finally, LSD increased Shannon's entropy, which represents the average rate at which information is produced by the participants (Shannon, 1948).

These changes are generally consistent with the psychotomimetic hypothesis; however, while both schizophrenic and manic psychosis are linked to reduced speech coherence, only the latter presents more recurrent speech graph structure with increased verbosity (Mota et al., 2012). Sanz et al. analyzed reference samples from bipolar patients to demonstrate the similarity with LSD-induced acute changes in speech. The entropic brain hypothesis put forward by Carhart-Harris and colleagues is a more general framework to interpret these findings (Carhart-Harris et al., 2014; Carhart-Harris, 2018). According to Carhart-Harris and colleagues, 5-HT2A receptor stimulation by psychedelics has a net scrambling or disorganizing effect on brain activity at multiple spatial and temporal scales, including those associated with perception, cognition, and the production of language. Conversely, antipsychotic medications show effects opposite to those predicted by the entropic brain hypothesis, leading to slower articulation rate, increased pausing, shorter utterances, and overall less information production rate (de Boer et al., 2020). These changes in language production are related to D2 receptor antagonism, the main pharmacological mechanism of action of antipsychotic drugs; however, future studies should assess the effects of 5-HT2A receptor 
antagonists (e.g. ketanserin) on non-semantic language features in the light of the entropic brain hypothesis. Also, Sanz et al. investigated LSD, a drug presenting biphasic effects with a gradual shift from serotonergic to dopaminergic action. Given that some of the strongest effects were seen close to the second interview (225 min post-infusion), a role of dopaminergic stimulation on speech disorganization cannot be ruled out (Marona-Lewicka and Nichols, 2007).

There is an interesting discrepancy between the results published by Sanz and colleagues and early studies of speech under the effects of LSD, which seemed to favor similarities between schizophrenic psychoses and the psychedelic state. Amarel and Cheek (1965) showed that LSD reduced the predictability of speech by means of impaired semantic coherence, but also reduced the total number of spoken words. It must be noted, however, that Amarel and Cheek investigated individuals suffering from alcoholism in a clinical setting, a population that could have experienced high levels of unattended anxiety during the experience. In contrast, Sanz et al. investigated healthy and motivated subjects with significant previous experience with psychedelic drugs, who willingly decided to participate in the experiment. As usual with psychedelics, the importance of context (i.e. the set and setting) in the interpretation of experimental results should not be underestimated (Carhart-Harris et al., 2018).

Natural speech produced during or immediately after the acute effects of psychedelics can also be used to compare 5-HT2A receptor activation with other altered states of consciousness, such as rapid eye movement (REM) sleep, the part of sleep characterized by long and vivid dream reports after awakening. Kraehenmann and colleagues instructed their subjects to produce verbal reports of a guided mental imagery task conducted 7 hours after treatment with LSD or a placebo (Kraehenmann et al., 2017). Afterwards, they manually scored the cognitive bizarreness of reports produced under both conditions, and found it was significantly increased for the LSD condition. High cognitive bizarreness is typical of dream reports, being indicative of the "strange, irrational and fanciful quality of REM sleep dream mentation, which is characterized by the presence of improbable or impossible imaginary events, characters, objects, thoughts or feelings" (Kraehenmann et al., 2017; Hobson et al., 1987); thus, its increase is consistent with the loss of semantic coherence reported by Sanz and colleagues. 


\section{Analysis of retrospective reports}

Before the development of psychometric questionnaires, spoken or written retrospective reports constituted the principal medium to communicate the nature of different drug-induced experiences. We find a well-known example in the following passage from "The Doors of Perception", by Aldous Huxley:

"Like the flowers, they glowed, when I looked at them, with brighter colors, a profounder significance. Red books, like rubies; emerald books; books bound in white jade; books of agate; of aquamarine, of yellow topaz; lapis lazuli books whose color was so intense, so intrinsically meaningful, that they seemed to be on the point of leaving the shelves to thrust themselves more insistently on my attention" (Huxley, 1954).

After returning to baseline, the author describes the effects of the drug, recalling in this case how certain aspects of visual perception (the colors and salience of books in shelves) were profoundly altered. We note that the semantic content of such a retrospective report constitutes a first approximation to the subjective effects elicited by the drug; thus, if the author wrote about altered color perception, dizziness and anxiety, we can suspect that the experience included at least some of these effects. Moreover, if two sets of reports stemming from the use of different drugs are similar in terms of their semantic content, we can hypothesize that the drug-induced experiences were similar as well. In other words, the semantic similarity of retrospective reports could be used as a measure of similarity between the reported experiences.

This approach was first applied by Coyle et al. to the corpus of the Erowid Experience Vaults (Coyle et al., 2012). This corpus contains reports for an ample variety of psychoactive compounds; also, some of the compounds are linked to a very large number of reports. Coyle and colleagues used supervised machine learning methods to successfully separate between reports of different drugs based on the associated vocabulary frequency vectors, showing that different families of compounds were linked to narratives with distinctive semantic content. Importantly, the preprocessing of the data included standard steps, such as removal of stopwords and lemmatization, but also the identification and removal of drug-specific terms and of terms that are generally related 
to drug use, since these features could allow a highly accurate yet trivial classification of the reports.

Sanz and colleagues applied a similar yet more sophisticated framework to investigate the relationship between the pharmacological mechanism of action of a widespread variety of drugs and the shared semantic content of their associated Erowid reports (Sanz et al., 2018). This approach included the preprocessing steps already implemented by Coyle et al., but then used LSA to tackle the issue of sparse term-document matrices. The bottom panel of Figure 2, based on this analysis, contains the unsupervised classification of several drugs based on the semantic similarity of their associated subjective reports. Briefly, the reduced-rank term-frequency matrix reconstructed using LSA was used to compute the correlation matrix $\mathrm{C}_{\mathrm{ij}}$, which has in its $\mathrm{i}, \mathrm{j}$ entry the correlation between the term frequency vectors of the $\mathrm{i}$-th and $\mathrm{j}$-th drugs in the corpus. This matrix was then used as the input of an unsupervised module detection algorithm (Blondel et al., 2008) capable of identifying drugs whose within-group semantic similarity was higher than their similarity with other similarly identified groups (note that the network representation in the bottom panel of Figure 2 only shows links between drugs whose reports have a particularly high semantic similarity). These modules of similar drugs (in the semantic sense) recapitulate known subjective effects and mechanisms of action; for instance, it is possible to find modules composed mostly of natural (module a) and synthetic (module b) psychedelic compounds. The same happens for modules composed of sedative drugs (modules $\mathrm{c}$ and $\mathrm{d}$ ). Overall, the unsupervised classification of drugs in terms of the semantic content respects traditional categories such as antidepressants and antipsychotics (most commonly, prescription drugs), psychedelics, dissociatives, entactogens, stimulants, and sedatives, among others. 
LSD

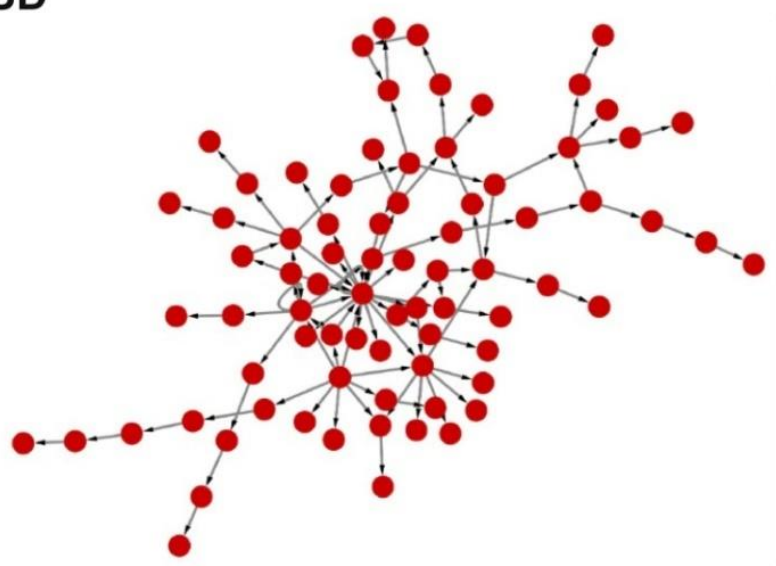

Antidepressants / antipsychotics



Placebo



Figure 2. Semantic and non-semantic analysis of reports produced during and after the psychedelic effects.

Top panel: Alterations in non-semantic features of speech produced under the acute effects of $75 \mu \mathrm{g}$ of LSD. Example graphs encoding speech produced under the acute effects of LSD (left) and under a placebo (right). Each node (red circles) corresponds to a word in the transcripts, and the directed links indicate that one word (target node) followed another (source node) at least once during the speech transcript (Sanz et al., 2021). Bottom panel: Semantic similarity network for reports of drug-induced experience (Sanz et al., 2018). Each node represents the combined Erowid's reports for a certain drug, colored according to 
categories based on their known effects and mechanism of action (see labels in the upper left). A connection between a pair of nodes indicates that the corresponding pair of reports present a sufficiently high degree of semantic similarity. The network was drawn using the ForceAtlas layout included in Gephi (https://gephi.org/), which tends to separate and identify groups of highly interconnected nodes that have sparser connections with the rest of the network (known as modules). Subpanels highlight different network modules, and the associated word clouds contain the name of drugs belonging to each module, with size weighted by their average strength of within module connections. These modules correspond to mostly natural and synthetic psychedelics (panels a and b, respectively), and to mostly natural and synthetic sedatives (panels c and d, respectively).

Interestingly, the comparison of subjective effects by means of retrospective reports is not limited to drug-induced experiences. As shown in Sanz et al. (2018), it is possible to construct a ranking of drugs in terms of the semantic similarity between their Erowid reports and dream reports, i.e. narratives about the content of individual dreams. Dreaming during sleep is a non-ordinary state of consciousness characterized by vivid multimodal imagery (ranging from elementary percepts to full-blown immersive hallucinations), altered sense of the relationship between the self and body boundaries, loss of the sense of agency, suppressed metacognitive function and heightened emotional reactivity (Nir and Tononi, 2010). According to anecdotal reports, these effects partially overlap with those elicited by psychedelic drugs, which has led several authors to speculate about a possible relationship between both states; however, the evidence directly supporting this overlap of effects is scarce (Snyder, 2001; Hobson, 2002; Carhart-Harris and Nutt, 2014; Kraehenmann, 2017). According to the large-scale analysis conducted in Sanz et al. (2018), psychedelics are the closest to dreams of high lucidity (i.e. when the dreamer is aware of being in the dreaming state; Voss et al., 2009) in terms of their induced subjective experience, with LSD being the closest across all drugs in the Erowid corpus. Consistently with their known subjective effects, experiences induced by deliriant compounds (e.g. those found in plants of the Datura genus) are the most similar to dreams of low lucidity. The use of LSA applied to retrospective subjective reports was also employed to demonstrate that ketamine, a glutamatergic dissociative agent, has subjective effects most similar to those reported after "near death experiences", i.e. episodes with real or perceived proximity to death that reliably include feelings of disconnection with the body (out of the body experiences) and of floating towards a light through a tunnel, a profound sense of bliss, a fast review of autobiographical memories, and the sense of an irreversible threshold (Martial et al., 
2019). Interestingly, the purported neuroprotective properties of ketamine (more precisely, its capacity to avoid cell death through over-excitation; Hudetz and Pagel, 2010) led to speculations of an endogenous chemical with a similar mechanism of action that is released at times critical for the survival of the organism; however, to date this compound remains to be identified (Jansen, 1997).

These studies foreshadow a possible research program to pursue a taxonomy of conscious states based on data-driven similarity metrics. Either implicitly or explicitly, most researchers operate under the assumption that consciousness can be graded along a unidimensional continuum, leading to the notion of "levels of consciousness". For instance, according to this idea, states such as coma or general anesthesia are "less conscious" than light sleep, which in turn is "less conscious" than ordinary wakefulness. Some of the conceptual problems with this notion have been highlighted by Bayne and colleagues (Bayne et al., 2016); in particular, the acute effects of serotonergic psychedelics are especially hard to reconcile with the idea of levels of consciousness (Bayne and Carter, 2018). While some popular accounts endorse the notion of the psychedelic state as an "expanded" (as opposed to diminished) state of consciousness, in reality psychedelics severely impair some aspects of human cognition and perception, while sparing or even facilitating others (Carter et al., 2005; Bouso et al., 2013; Bayne and Carter, 2018; Pokorny et al., 2020; Healy, 2021). Taking these considerations into account, several independent dimensions are likely to be required to fully characterize the repertoire of conscious states, yet disagreements exist about what these dimensions should be.

The type of analysis represented in Figure 2 (bottom panel) might represent an interim solution to this open problem: it is not necessary to find the dimensions needed for the adequate description and characterization of conscious states if one is capable of estimating their pairwise similarity. Armed with this information, it is possible to classify states of consciousness based on their similarity; moreover, their hierarchical clustering (i.e. finding clusters within clusters) could allow the identification of conscious states at different levels of temporal granularity. This is perhaps the main issue regarding the difficulties faced by attempts to precisely define states of consciousness (Tassi and Muzet, 2001). For instance, does an orgasm qualify as such? What about a deja vu, falling in love, or a near-death experience? All these examples include a transient but stable 
modification in the quality of conscious experience, yet some of them might be considered too fleeting to qualify as conscious states. In reality, however, this a problem of resolution, i.e. how deep we want to look into the hierarchical modular structure of similarity networks such as the one depicted in Figure 2 (bottom panel).

While the similarity between the subjective experience of different conscious states could also be inferred from the comparison between psychometric questionnaires, without knowing the relevant dimensions necessary to capture the variability of states it does not seem possible to design the adequate questionnaires in the first place. The computational analysis of retrospective narratives seems to offer a data-driven alternative to this conundrum, since it allows to estimate the similarity of effects based on the semantic distance between reports, without constraining the participants to answer a predefined set of questions.

\section{Semantic similarity parallels neurochemical and pharmacological similarity}

What is the relationship between the mechanism of action of different drugs and the subjective effects they elicit? This is perhaps one of the most difficult yet fascinating questions in consciousness research. Drug-induced experiences are well-characterized at the molecular and cellular levels; for instance, displacement assays with radiolabeled compounds allow the in vitro estimation of drug affinities for specific binding sites, which include most of the major neurotransmitter receptors types and sub-types (Bylund and Toews, 1993). This information can be summarized in drug-specific affinity profiles. Also, more information is available for certain drugs and receptors, including functional assays (informing whether the drug behaves as an agonist, antagonist, or inverse agonist), changes in gene expression (González-Maeso et al., 2003), and second messenger recruitment in G-protein coupled receptors (GPCR; López-Giménez et al., 2017). However, in spite of this characterization at the molecular scale, little is known about the downstream effects on large-scale activity patterns that correlate with cognition and conscious experience and how these effects depend on the molecular level.

Recent work by Zamberlan and colleagues pursued the following hypothesis: if the subjective experience of psychedelic drugs is determined by their action at the molecular level, then the closer two drugs are at this level (e.g. the more similar their binding affinity profiles), then the closer 
those drugs should be in terms of the semantic content of their associated subjective reports (Zamberlan et al., 2018). The scatter plot presented in Figure 3A supports this hypothesis, showing a positive correlation $(\mathrm{R}=0.6)$ between pairwise semantic similarity and the similarity of binding affinity profiles (compare also the binding affinity and semantic similarity matrices in the same panel). This result is particularly striking given it was obtained from a group of drugs that were, for the most part, serotonergic psychedelics (Ray, 2010). It is well established that these compounds act due to their affinity to 5-HT2A receptors (Nichols, 2016), yet if this is the only relevant mechanism of action, how can different psychedelic drugs present a variety of different effects? A candidate solution is explored by Zamberlan and colleagues: while 5-HT2A agonism is a necessary condition for psychedelic activity, the action of the drug at other binding sites can nuance the resulting subjective effects (note that this hypothesis is not mutually exclusive with the possibility of functional selectivity at GPCR, such as the 5-HT2A receptor; Nichols, 2017).

The relationship between subjective effects and neurochemical action of psychedelic compounds can be further informed by natural language processing applied to subjective reports. Suppose the binding affinity similarity between two compounds is not computed by comparing their affinities across all assayed receptors, but instead restricted to a subset of binding sites. In this case, if the correlation between binding affinity and semantic similarities increases, it could be concluded that that activity at this subset of receptors is more specific to determine the subjective effects of the compound. This idea is exploited in the analysis shown in Figure 3B, showing the correlation between semantic and affinity similarities computed with the binding affinity restricted to several possible combinations of receptor subsets. The top subpanel of Figure 3B, extracted from Zamberlan et al. (2018), shows the obtained correlation values, sorted in ascending order, while the lower panel highlights in yellow whether the receptor families in the rows were considered for the computation of the binding affinity similarities (these receptor families include serotonergic, dopaminergic, adrenergic, histaminergic, glutamatergic and cannabinoid receptors, plus transporter proteins and $\mathrm{Ca}+$ channels, among other groups of binding sites). 


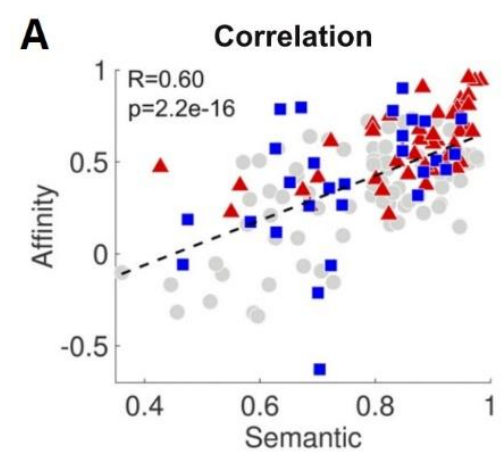

B



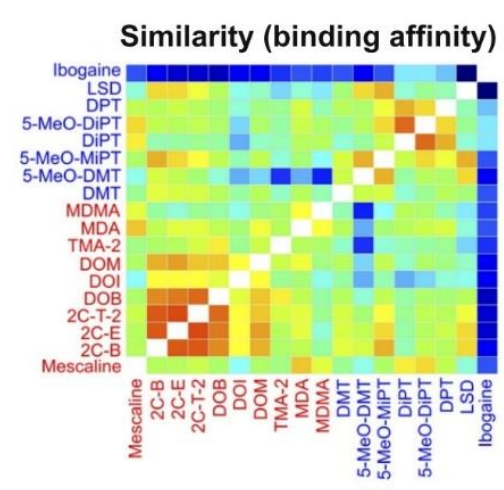

C

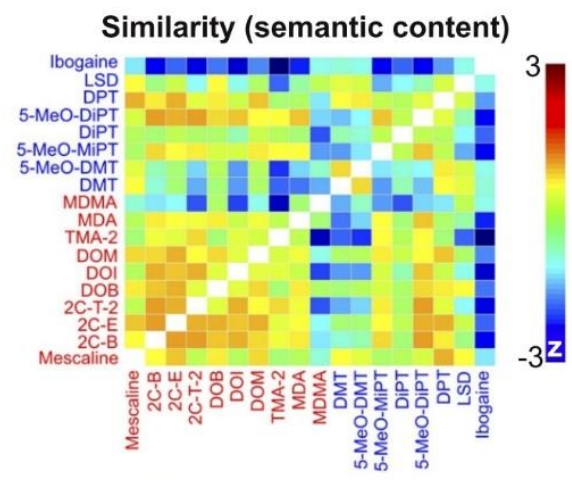

D

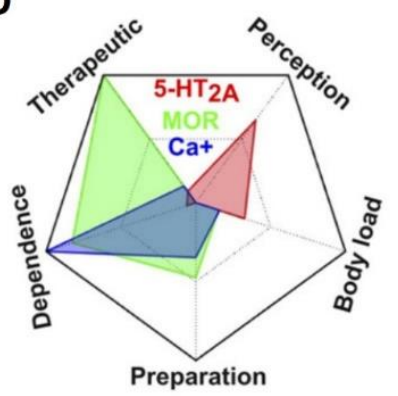

Figure 3. The semantic similarity of subjective reports is correlated with the similarity of binding affinity profiles for 18 psychedelic tryptamines and phenethylamines (panel A, left), from Zamberlan et al., 2018. This can be appreciated by direct visual inspection of the corresponding similarity matrices (panel A, right).

Panel B shows the correlation between semantic and pharmacological similarity matrices, with the latter computed using information from different receptor subsets, allowing to identify an optimal group of receptors to explain the semantic content of the reports. The principal components of the reduced ranked term-document matrix represent recurrent topics in the corpus, with variable prevalence among the psychedelic drugs under study (panel C). This type of analysis is also capable of linking affinity to certain receptors with specific topics (panel D), e.g. 5-HT2A with the "perception" topic, or MOR with the "dependence" and "therapeutic" topics.

Taking this analysis one step further, it is possible to decompose the subjective reports into topics, given by sets of terms that tend to co-occur through the documents, summarizing certain repeating themes. Figure 3D lists some topics obtained by Zamberlan and colleagues using the reports associated with several psychedelic tryptamines and phenethylamines, which include topics such as "changes to visual perception", "body load", "drug preparation", "drug dependency" and "therapeutic use"; this panel also presents the prevalence of each topic (columns) across the drugs 
(rows). By relating topics and affinities it is possible to inform the subjective effects linked to high binding affinity at different sites, as shown in Figure 3E for three example receptors (note that high affinity for 5-HT2A indicates the prevalence of the "changes to visual perception" topic, as expected). The idea of linking receptor activation with subjective effects through NLP applied to retrospective reports has been further exploited by Ballentine and colleagues to map brain areas whose neurochemical stimulation underlies specific subjective experiences (Ballentine et al., 2011). This work related the semantic content of over 6.000 narratives of drug-induced experiences with the binding affinity at 40 neurotransmitter receptor subtypes, which were mapped to 3D coordinates in the brain via their gene transcription levels from invasive tissue probes.

The process by which psychedelic drugs elicit their effects spreads across brain scales, from the molecular level to the large-scale patterns of coordinated activity correlating with different cognitive functions and contents of conscious experience. At the bottom of the hierarchy, small molecules (drugs) interact with proteins (receptors) and this interaction can be described (among other metrics) by the binding affinity between both. Next, for GPCR, different second messengers can be recruited depending on the final shape of the ligand-receptor complex; these downstream effects can determine functional consequences in terms of neural dynamics (e.g. cell excitation or inhibition). In turn, neurons interact through synapses and form networks that extend through cortical and sub-cortical areas; it is widely believed that the neuroelectrical configuration of these neural networks is the main substrate on which conscious experience depends. Thus, at the highest level of this hierarchy, subjective experience is modified by psychedelic action, which can be assessed by means of oral or written natural language reports.

Within each level, we find elements that allow the introduction of a metric of similarity between compounds, ranging from the molecular similarity between drugs to the semantic similarity between drug-induced narratives. Thus, the analysis conducted in Zamberlan et al. (2018) could be replicated at multiple scales once the necessary empirical data becomes available, with the expectation that the correlation between semantic similarities and those obtained using data at each other level increases with the proximity to the top of the hierarchy, e.g. that similarity between evoked large-scale activity correlates better with the semantic similarity of subjective reports than the similarity between binding affinity profiles, which in turn does it better than the structural 
similarity between molecules. However, note that this expectation might not be fulfilled if measurements become noisier or less reliable at the higher levels - for instance, consider the limitations of indirect brain measuring techniques such as fMRI or PET.

\section{Natural language reports and the therapeutic use of psychedelics}

After decades of little to none research, an ongoing surge of studies has demonstrated the potential usefulness of psychedelics to treat psychiatric disorders such as depression and anxiety (Sessa, 2005; Nutt and Carhart-Harris, 2021; Mertens and Preller, 2021; Yaden et al., 2021). In contrast to current pharmacological treatments, the subjective effects induced by psychedelics appear to influence the outcome of the intervention - for instance, current evidence suggests that patients who undergo mystical-type experiences evolve comparatively better after drug intake (Griffiths et al., 2016). Considering the potential relevance of conscious experience for the therapeutic action of psychedelics, it is reasonable to hypothesize that natural language reports carry relevant information to determine the success and predict the outcome of interventions using these compounds.

This possibility has been first explored by Carrillo and colleagues, who showed that baseline interview data can predict which patients will respond to psilocybin for treatment-resistant depression (Carrillo et al., 2018). In this study, quantitative metrics related to the emotional content of the interviews were extracted and used as input to train machine learning algorithms, which were then used to identify responders from non-responders with significant ( 80\%) accuracy.

The conceptual framework introduced in this study was further developed by Cox and colleagues, and applied it to a much larger dataset of natural language narratives to determine who among more than 1000 individuals would quit or reduce using drugs following a psychedelic experience (Cox et al., 2021). Here, the features for the machine learning analysis were based on the semantic content of the narratives, more precisely on the prevalence of different topics. While the accuracy was below the value reported by Carrillo and colleagues, it nevertheless reached statistical significance $(\sim 60 \%)$, showing that natural language reports obtained from a large sample of individuals have information relevant for the prediction of therapeutic outcomes. 
The use of natural language processing in the incipient field of psychedelic-assisted therapy is not an isolated trend. In recent years it has become evident that the data fingerprint produced by an individual contains information relevant for the early diagnosis and prognosis of several neuropsychiatric disorders (Meyer-Lindenberg, 2018; Sigman et al., 2021). Speech produced under natural or ecological conditions is perhaps among the most useful streams of data that can be tapped for automated machine learning models of mental health, since it is produced in large amounts, it is cheap to obtain and analyze, and might reflect the emotional content of the speakers, as well as their ongoing thought processes (Garfield et al., 1992; Le Glaz et al., 2021). Based on these examples, we can speculate that the automated analysis of natural speech will consolidate into a valuable tool to assist the decision-making process of clinicians, and to optimize the design and implementation of therapy sessions assisted by psychedelic compounds, with the objective of maximizing therapeutic gain and reducing the likelihood of anxiety in the patients. Because of this, we recommend that future clinical studies include the recording of natural speech samples before, during and after the acute effects of psychedelic compounds.

\section{Limitations and future directions}

We have reviewed several key studies illustrating how the analysis of written or spoken natural language reports can assist the investigation of the subjective effects and mechanisms of action of psychedelic compounds. The usefulness of this approach, however, must be considered within the context of several limitations, some of them intrinsic to the acquisition and analysis of natural language.

The most obvious limitation of this approach is also related to one of its main potential advantages: the unconstrained nature of natural language reports. When subjects speak freely about a certain subject (for instance, about their subjective experience with a psychedelic compound) they might omit important details or fail to clearly express the most relevant information. Additionally, some subjects might be better communicators than others; their reports are then expected to be more informative in comparison. Since they are participating in a scientific experiment and being interviewed by a specialist, subjects might incorrectly assume that certain information is implicit in the conversation and thus fail to include it in their reports. Moreover, some personality traits of 
the participants could be manifest in their reports, confounding attempts to objectivize their content using automated methods. In contrast, standardized psychometric questionnaires have the advantage of uniformly guiding the participants through the points that are considered most relevant by the researchers. Overcoming this limitation depends on training the interviewers to obtain the most of the participants and their reports, for instance, by guiding them through a series of predefined questions, or by bringing them back on track in case they begin to digress. One prominent example of this procedure can be found in the microphenomenological interview (Petitmengin et al., 2019). Interviewers adopting this method are trained to identify the main contents of the narrated experience, to assist the subjects in their evocation process, to detect and document implicit information, and to assist the subjects in the process of formulating their reports, among other precautions.

The analysis of large online databases (e.g. Erowid's Experience Vaults) presents additional problems associated with unknown or underinformed variables, such as the precise nature of the compounds that were consumed, their dosage, whether drugs were consumed alone or in combination with others, subject demographics, mental health status and past history of drug use. Furthermore, except for relatively recent projects (see for example https://effectindex.com/), ongoing efforts for the collection of drug use reports query participants about their experiences using a single text box, and thus fail to distinguish between preparation, set and setting, the acute phase of the experience, and its short and long term consequences. While some of these problems could be overcome by imposing more structure to the reports (e.g. forcing participants to narrate separately different aspects of the experience), other limitations (such as insufficient or unreliable information concerning drugs) are more difficult to solve. In those cases, it should be determined whether the availability of large amounts of data can compensate for the potential noise introduced by these limitations.

In spite of these potential shortcomings, the analysis of natural language reports can still be considered a promising tool to tackle research questions about the nature of subjective experience, in particular, those about effects and neurochemical action of serotonergic psychedelics. When finally answered, some of the following questions will serve to more precisely outline the 
advantages and limitations of natural language over more traditional and established methodologies.

Is it possible to infer the score of psychometric scales, such as 5D-ASC, from unconstrained reports? This question could be answered at the level of individual reports, or at the level of the average scores obtained for a given drug.

Is it possible to accurately estimate the dosage of a drug based on reports obtained during the acute effects, or based on retrospective reports obtained after return to baseline? In general, which of these two approaches conveys more information about this and other facets of the experience?

Can we disentangle the contents of a report from the individual traits of the reporter? How are these traits manifest in a typical report? What is the effect of language, culture, social status, age, gender, etc., on the way participants express their drug-induced experiences?

When used to predict the outcome of psychedelic treatments, how does the accuracy obtained using natural language reports compare with that obtained using questionnaires? In case the accuracy is significantly higher, is it possible to identify the information that is conveyed by natural language reports that is left out of more structured assessments?

How is the semantic similarity between drug use reports related to other metrics of similarity between drugs and their effects on the brain? At which level is the semantic content of the reports optimally represented?

What is the temporal resolution of retrospective narratives? How much temporally ordered information about subjective experience can be conveyed by means of natural language reports, and how is this information distorted?

\section{Conclusions}

Language is our main everyday vehicle for the expression of ideas, emotions, plans, and subjective, inner feelings. After experiencing the acute effects of a psychedelic drug, many individuals feel a 
strong urge to communicate with others and exchange impressions about what happened and how it felt. Taken together, the articles we reviewed serve to demonstrate how this urge can be leveraged for the scientific exploration of serotonergic psychedelics. While the merits of natural language processing over more traditional methods to inquire about subjective experiences remain to be demonstrated, we nevertheless encourage researchers to acquire natural language samples in their experiments, and to address some of the open questions that will ultimately determine the usefulness of this approach.

\section{Acknowledgments}

The author thanks Camila Sanz for her assistance with Figure 2. This work was supported by a Mercator fellowship funded by the German Research Foundation, and by grant PICT-2019-02294 funded by Agencia I+D+I (Argentina).

\section{References}

Amarel, M., \& Cheek, F. E. (1965). Some effects of LSD-25 on verbal communication. Journal of Abnormal Psychology, 70(6), 453.

Ballentine, G., Friedman, S. F., \& Bzdok, D. (2021). Trips and Neurotransmitters: Discovering Principled Patterns across 6,850 Hallucinogenic Experiences. bioRxiv.

Barrett, F. S., Johnson, M. W., \& Griffiths, R. R. (2015). Validation of the revised Mystical Experience Questionnaire in experimental sessions with psilocybin. Journal of Psychopharmacology, 29(11), 1182-1190.

Barrett, F. S., Bradstreet, M. P., Leoutsakos, J. M. S., Johnson, M. W., \& Griffiths, R. R. (2016). The Challenging Experience Questionnaire: Characterization of challenging experiences with psilocybin mushrooms. Journal of Psychopharmacology, 30(12), 1279-1295.

Bayne, T., Hohwy, J., \& Owen, A. M. (2016). Are there levels of consciousness?. Trends in cognitive sciences, 20(6), 405-413. 
Bayne, T., \& Carter, O. (2018). Dimensions of consciousness and the psychedelic state. Neuroscience of consciousness, $2018(1)$, niy008.

Bedi, G., Carrillo, F., Cecchi, G. A., Slezak, D. F., Sigman, M., Mota, N. B., ... \& Corcoran, C. M. (2015). Automated analysis of free speech predicts psychosis onset in high-risk youths. npj Schizophrenia, 1(1), 1-7.

Bertola, L., Mota, N. B., Copelli, M., Rivero, T., Diniz, B. S., Romano-Silva, M. A., ... \& Malloy-Diniz, L. F. (2014). Graph analysis of verbal fluency test discriminate between patients with Alzheimer's disease, mild cognitive impairment and normal elderly controls. Frontiers in aging neuroscience, $6,185$.

Blondel, V. D., Guillaume, J. L., Lambiotte, R., \& Lefebvre, E. (2008). Fast unfolding of communities in large networks. Journal of statistical mechanics: theory and experiment, 2008(10), P10008.

Bouso, J. C., Fábregas, J. M., Antonijoan, R. M., Rodríguez-Fornells, A., \& Riba, J. (2013). Acute effects of ayahuasca on neuropsychological performance: differences in executive function between experienced and occasional users. Psychopharmacology, 230(3), 415-424.

Bullmore, E., \& Sporns, O. (2009). Complex brain networks: graph theoretical analysis of structural and functional systems. Nature reviews neuroscience, 10(3), 186-198.

Bylund, D. B., \& Toews, M. L. (1993). Radioligand binding methods: practical guide and tips. American Journal of Physiology-Lung Cellular and Molecular Physiology, 265(5), L421-L429.

Carhart-Harris, R. L., Erritzoe, D., Williams, T., Stone, J. M., Reed, L. J., Colasanti, A., ... \& Nutt, D. J. (2012). Neural correlates of the psychedelic state as determined by fMRI studies with psilocybin. Proceedings of the National Academy of Sciences, 109(6), 2138-2143. 
Carhart-Harris, R. L., Leech, R., Hellyer, P. J., Shanahan, M., Feilding, A., Tagliazucchi, E., ... \& Nutt, D. (2014). The entropic brain: a theory of conscious states informed by neuroimaging research with psychedelic drugs. Frontiers in human neuroscience, 8, 20.

Carhart-Harris, R., \& Nutt, D. (2014). Was it a vision or a waking dream?. Frontiers in psychology, 5, 255.

Carhart-Harris, R. L. (2018). The entropic brain-revisited. Neuropharmacology, 142, 167-178.

Carhart-Harris, R. L., Roseman, L., Haijen, E., Erritzoe, D., Watts, R., Branchi, I., \& Kaelen, M. (2018). Psychedelics and the essential importance of context. Journal of Psychopharmacology, $32(7), 725-731$.

Carhart-Harris, R. L., Muthukumaraswamy, S., Roseman, L., Kaelen, M., Droog, W., Murphy, K., ... \& Nutt, D. J. (2016). Neural correlates of the LSD experience revealed by multimodal neuroimaging. Proceedings of the National Academy of Sciences, 113(17), 4853-4858.

Carrillo, F., Sigman, M., Slezak, D. F., Ashton, P., Fitzgerald, L., Stroud, J., ... \& Carhart-Harris, R. L. (2018). Natural speech algorithm applied to baseline interview data can predict which patients will respond to psilocybin for treatment-resistant depression. Journal of affective disorders, 230, 84-86.

Carter, O. L., Burr, D. C., Pettigrew, J. D., Wallis, G. M., Hasler, F., \& Vollenweider, F. X. (2005). Using psilocybin to investigate the relationship between attention, working memory, and the serotonin 1A and 2A receptors. Journal of Cognitive neuroscience, 17(10), 1497-1508.

Chowdhury, G. G. (2003). Natural language processing. Annual review of information science and technology, 37(1), 51-89. 
Coelho, R. M., Drummond, C., Mota, N. B., Erthal, P., Bernardes, G., Lima, G., ... \& Mattos, P. (2021). Network analysis of narrative discourse and attention-deficit hyperactivity symptoms in adults. Plos one, 16(4), e0245113.

Corcoran, C. M., Carrillo, F., Fernández-Slezak, D., Bedi, G., Klim, C., Javitt, D. C., ... \& Cecchi, G. A. (2018). Prediction of psychosis across protocols and risk cohorts using automated language analysis. World Psychiatry, 17(1), 67-75.

Cox, D. J., Garcia-Romeu, A., \& Johnson, M. W. (2021). Predicting changes in substance use following psychedelic experiences: natural language processing of psychedelic session narratives. The American Journal of Drug and Alcohol Abuse, 1-11.

Coyle, J. R., Presti, D. E., \& Baggott, M. J. (2012). Quantitative analysis of narrative reports of psychedelic drugs. arXiv preprint arXiv:1206.0312.

de Boer, J. N., Voppel, A. E., Brederoo, S. G., Wijnen, F. N. K., \& Sommer, I. E. C. (2020). Language disturbances in schizophrenia: the relation with antipsychotic medication. NPJ schizophrenia, 6(1), 1-9.

Dittrich, A. (1998). The standardized psychometric assessment of altered states of consciousness (ASCs) in humans. Pharmacopsychiatry, 31(S 2), 80-84.

Elvevåg, B., Foltz, P. W., Weinberger, D. R., \& Goldberg, T. E. (2007). Quantifying incoherence in speech: an automated methodology and novel application to schizophrenia. Schizophrenia research, 93(1-3), 304-316.

Escohotado, A. (1999). A brief history of drugs: From the stone age to the stoned age. Simon and Schuster. 
Family, N., Vinson, D., Vigliocco, G., Kaelen, M., Bolstridge, M., Nutt, D. J., \& Carhart-Harris, R. L. (2016). Semantic activation in LSD: evidence from picture naming. Language, Cognition and Neuroscience, 31(10), 1320-1327.

Garfield, D. A., Rapp, C., \& Evens, M. (1992). Natural language processing in psychiatry: Artificial intelligence technology and psychopathology. Journal of Nervous and Mental Disease.

Gonçalves, F. L. (2021). Basic Concepts of Behavioral Pharmacology. In Behavior Analysis and Substance Dependence (pp. 39-49). Springer, Cham.

González-Maeso, J., Yuen, T., Ebersole, B. J., Wurmbach, E., Lira, A., Zhou, M., ... \& Sealfon, S. C. (2003). Transcriptome fingerprints distinguish hallucinogenic and nonhallucinogenic 5hydroxytryptamine $2 \mathrm{~A}$ receptor agonist effects in mouse somatosensory cortex. Journal of Neuroscience, 23(26), 8836-8843.

Griffiths, R. R., Johnson, M. W., Carducci, M. A., Umbricht, A., Richards, W. A., Richards, B. D., ... \& Klinedinst, M. A. (2016). Psilocybin produces substantial and sustained decreases in depression and anxiety in patients with life-threatening cancer: A randomized double-blind trial. Journal of psychopharmacology, 30(12), 1181-1197.

Hanks, J. B., \& González-Maeso, J. (2013). Animal models of serotonergic psychedelics. ACS chemical neuroscience, 4(1), 33-42.

Healy, C. J. (2021). The acute effects of classic psychedelics on memory in humans. Psychopharmacology, 1-15.

Hobson, J. A., Hoffman, S. A., Helfand, R., \& Kostner, D. (1987). Dream bizarreness and the activation-synthesis hypothesis. Human neurobiology.

Hobson, J. A. (2002). The dream drugstore: Chemically altered states of consciousness. MIT Press. 
Hudetz, J. A., \& Pagel, P. S. (2010). Neuroprotection by ketamine: a review of the experimental and clinical evidence. Journal of cardiothoracic and vascular anesthesia, 24(1), 131-142.

Huxley, A. (1954). The doors of perception and Heaven and hell. Random House.

Iter, D., Yoon, J., \& Jurafsky, D. (2018, June). Automatic detection of incoherent speech for diagnosing schizophrenia. In Proceedings of the Fifth Workshop on Computational Linguistics and Clinical Psychology: From Keyboard to Clinic (pp. 136-146).

Jansen, K. L. (1997). The ketamine model of the near-death experience: A central role for the Nmethyl-D-aspartate receptor. Journal of Near-Death Studies, 16(1), 5-26.

Johnson, M. W., Hendricks, P. S., Barrett, F. S., \& Griffiths, R. R. (2019). Classic psychedelics: An integrative review of epidemiology, therapeutics, mystical experience, and brain network function. Pharmacology \& therapeutics, 197, 83-102.

Kettner, H., Gandy, S., Haijen, E. C., \& Carhart-Harris, R. L. (2019). From egoism to ecoism: Psychedelics increase nature relatedness in a state-mediated and context-dependent manner. International journal of environmental research and public health, 16(24), 5147.

Klema, V., \& Laub, A. (1980). The singular value decomposition: Its computation and some applications. IEEE Transactions on automatic control, 25(2), 164-176.

Kometer, M., Pokorny, T., Seifritz, E., \& Volleinweider, F. X. (2015). Psilocybin-induced spiritual experiences and insightfulness are associated with synchronization of neuronal oscillations. Psychopharmacology, 232(19), 3663-3676.

Kraehenmann, R., Pokorny, D., Vollenweider, L., Preller, K. H., Pokorny, T., Seifritz, E., \& Vollenweider, F. X. (2017). Dreamlike effects of LSD on waking imagery in humans depend on serotonin 2A receptor activation. Psychopharmacology, 234(13), 2031-2046. 
Kraehenmann, R. (2017). Dreams and psychedelics: neurophenomenological comparison and therapeutic implications. Current neuropharmacology, 15(7), 1032-1042.

Landauer, T. K., Foltz, P. W., \& Laham, D. (1998). An introduction to latent semantic analysis. Discourse processes, 25(2-3), 259-284.

Le Glaz, A., Haralambous, Y., Kim-Dufor, D. H., Lenca, P., Billot, R., Ryan, T. C., ... \& Lemey, C. (2021). Machine learning and natural language processing in mental health: Systematic review. Journal of Medical Internet Research, 23(5), e15708.

López-Giménez, J. F., \& González-Maeso, J. (2017). Hallucinogens and serotonin 5-HT 2A receptor-mediated signaling pathways. Behavioral Neurobiology of Psychedelic Drugs, 45-73.

Marona-Lewicka, D., \& Nichols, D. E. (2007). Further evidence that the delayed temporal dopaminergic effects of LSD are mediated by a mechanism different than the first temporal phase of action. Pharmacology Biochemistry and Behavior, 87(4), 453-461.

Marggraf, M. P., Cohen, A. S., Davis, B. J., DeCrescenzo, P., Bair, N., \& Minor, K. S. (2018). Semantic coherence in psychometric schizotypy: an investigation using latent semantic analysis. Psychiatry research, 259, 63-67.

Martial, C., Cassol, H., Charland-Verville, V., Pallavicini, C., Sanz, C., Zamberlan, F., ... \& Tagliazucchi, E. (2019). Neurochemical models of near-death experiences: a large-scale study based on the semantic similarity of written reports. Consciousness and cognition, 69, 52-69.

Mertens, L. J., \& Preller, K. H. (2021). Classical Psychedelics as Therapeutics in PsychiatryCurrent Clinical Evidence and Potential Therapeutic Mechanisms in Substance Use and Mood Disorders. Pharmacopsychiatry, 49.

Meyer-Lindenberg, A. (2018). Artificial intelligence in psychiatry-an overview. Der Nervenarzt, 89(8), 861-868. 
Mikolov, T., Chen, K., Corrado, G., \& Dean, J. (2013). Efficient estimation of word representations in vector space. arXiv preprint arXiv:1301.3781.

Mota, N. B., Vasconcelos, N. A., Lemos, N., Pieretti, A. C., Kinouchi, O., Cecchi, G. A., ... \& Ribeiro, S. (2012). Speech graphs provide a quantitative measure of thought disorder in psychosis. PloS one, 7(4), e34928.

Mota, N. B., Furtado, R., Maia, P. P., Copelli, M., \& Ribeiro, S. (2014). Graph analysis of dream reports is especially informative about psychosis. Scientific reports, 4(1), 1-7.

Mota, N. B., Copelli, M., \& Ribeiro, S. (2017). Thought disorder measured as random speech structure classifies negative symptoms and schizophrenia diagnosis 6 months in advance. $n p j$ Schizophrenia, 3(1), 1-10.

Muthukumaraswamy, S. D., Carhart-Harris, R. L., Moran, R. J., Brookes, M. J., Williams, T. M., Errtizoe, D., ... \& Nutt, D. J. (2013). Broadband cortical desynchronization underlies the human psychedelic state. Journal of Neuroscience, 33(38), 15171-15183.

Nichols, D. E. (2016). Psychedelics. Pharmacological reviews, 68(2), 264-355.

Nichols, D. E. (2017). Chemistry and structure-activity relationships of psychedelics. Behavioral Neurobiology of Psychedelic Drugs, 1-43.

Nichols, D. E., \& Walter, H. (2020). The history of psychedelics in psychiatry. Pharmacopsychiatry.

Nir, Y., \& Tononi, G. (2010). Dreaming and the brain: from phenomenology to neurophysiology. Trends in cognitive sciences, 14(2), 88-100. 
Nour, M. M., Evans, L., Nutt, D., \& Carhart-Harris, R. L. (2016). Ego-dissolution and psychedelics: validation of the ego-dissolution inventory (EDI). Frontiers in human neuroscience, 10, 269.

Nutt, D., \& Carhart-Harris, R. (2021). The current status of psychedelics in psychiatry. JAMA psychiatry, 78(2), 121-122.

Pallavicini, C., Cavanna, F., Zamberlan, F., de la Fuente, L. A., Arias, M., Romero, M. C., ... \& Tagliazucchi, E. (2020). Neural and subjective effects of inhaled DMT in natural settings. $J$ Psychopharmacol (in press)

Petitmengin, C., van Beek, M., Bitbol, M., Nissou, J. M., \& Roepstorff, A. (2019). Studying the experience of meditation through micro-phenomenology. Current opinion in psychology, 28, 5459.

Preller, K. H., \& Vollenweider, F. X. (2016). Phenomenology, structure, and dynamic of psychedelic states. Behavioral neurobiology of psychedelic drugs, 221-256.

Preller, K. H., Burt, J. B., Ji, J. L., Schleifer, C. H., Adkinson, B. D., Stämpfli, P., ... \& Anticevic, A. (2018). Changes in global and thalamic brain connectivity in LSD-induced altered states of consciousness are attributable to the 5-HT2A receptor. Elife, 7, e35082.

Pokorny, T., Duerler, P., Seifritz, E., Vollenweider, F. X., \& Preller, K. H. (2020). LSD acutely impairs working memory, executive functions, and cognitive flexibility, but not risk-based decision-making. Psychological medicine, 50(13), 2255-2264.

Ray, T. S. (2010). Psychedelics and the human receptorome. PloS one, 5(2), e9019.

Roseman, L., Haijen, E., Idialu-Ikato, K., Kaelen, M., Watts, R., \& Carhart-Harris, R. (2019). Emotional breakthrough and psychedelics: validation of the emotional breakthrough inventory. Journal of Psychopharmacology, 33(9), 1076-1087. 
Sahlgren, M. (2006). The Word-Space Model: Using distributional analysis to represent syntagmatic and paradigmatic relations between words in high-dimensional vector spaces (Doctoral dissertation, Institutionen för lingvistik).

Sanz, C., Zamberlan, F., Erowid, E., \& Tagliazucchi, E. (2018). The experience elicited by hallucinogens presents the highest similarity to dreaming within a large database of psychoactive substance reports. Frontiers in neuroscience, 12, 7.

Sanz, C., Pallavicini, C., Carrillo, F., Zamberlan, F., Sigman, M., Mota, N., ... \& Tagliazucchi, E. (2021). The entropic tongue: Disorganization of natural language under LSD. Consciousness and Cognition, 87, 103070.

Samorini, G. (2002). Animals and psychedelics: The natural world and the instinct to alter consciousness. Simon and Schuster.

Saulin, A., Savli, M., \& Lanzenberger, R. (2012). Serotonin and molecular neuroimaging in humans using PET. Amino acids, 42(6), 2039-2057.

Schmidt, T. T., \& Berkemeyer, H. (2018). The altered states database: Psychometric data of altered states of consciousness. Frontiers in psychology, 9, 1028.

Sessa, B. (2005). Can psychedelics have a role in psychiatry once again?. The British Journal of Psychiatry, 186(6), 457-458.

Shannon, C. E. (1948). A mathematical theory of communication. The Bell system technical journal, 27(3), 379-423.

Sigman, M., Slezak, D. F., Drucaroff, L., Ribeiro, S., \& Carrillo, F. (2021). Artificial and Human Intelligence in Mental Health. AI Magazine, 42(1), 39-46. 
Snyder, S. H. (2001). Psychedelics, psychosis and dreaming. nature neuroscience, 4(6), 563-563.

Spencer, T. J., Thompson, B., Oliver, D., Diederen, K., Demjaha, A., Weinstein, S., ... \& McGuire, P. (2021). Lower speech connectedness linked to incidence of psychosis in people at clinical high risk. Schizophrenia Research, 228, 493-501.

Studerus, E., Gamma, A., \& Vollenweider, F. X. (2010). Psychometric evaluation of the altered states of consciousness rating scale (OAV). PloS one, 5(8), e12412.

Tagliazucchi, E., Roseman, L., Kaelen, M., Orban, C., Muthukumaraswamy, S. D., Murphy, K., ... \& Carhart-Harris, R. (2016). Increased global functional connectivity correlates with LSD-induced ego dissolution. Current Biology, 26(8), 1043-1050.

Tagliazucchi, E., Zamberlan, F., Cavanna, F., de la Fuente, L. A., Romero, C., Perl, Y. S., \& Pallavicini, C. (2021). Baseline power of theta oscillations predicts mystical-type experiences induced by DMT. Frontiers in Psychiatry (in press).

Tassi, P., \& Muzet, A. (2001). Defining the states of consciousness. Neuroscience \& Biobehavioral Reviews, 25(2), 175-191.

Timmermann, C., Roseman, L., Schartner, M., Milliere, R., Williams, L. T., Erritzoe, D., ... \& Carhart-Harris, R. L. (2019). Neural correlates of the DMT experience assessed with multivariate EEG. Scientific reports, 9(1), 1-13.

Voss, U., Holzmann, R., Tuin, I., \& Hobson, A. J. (2009). Lucid dreaming: a state of consciousness with features of both waking and non-lucid dreaming. Sleep, 32(9), 1191-1200.

Wießner, I., Falchi, M., Palhano-Fontes, F., Oliveira Maia, L., Feilding, A., Ribeiro, S., ... \& Tófoli, L. F. (2021). Low-dose LSD and the stream of thought: Increased Discontinuity of Mind, Deep Thoughts and abstract flow. Psychopharmacology, 1-13.

Wittgenstein, L. (1953). Philosophical investigations. John Wiley \& Sons. 
Yaden, D. B., Yaden, M. E., \& Griffiths, R. R. (2021). Psychedelics in Psychiatry-Keeping the Renaissance From Going Off the Rails. JAMA Psychiatry, 78(5), 469-470.

Yarkoni, T., Poldrack, R. A., Nichols, T. E., Van Essen, D. C., \& Wager, T. D. (2011). Largescale automated synthesis of human functional neuroimaging data. Nature methods, 8(8), 665670.

Zamberlan, F., Sanz, C., Martínez Vivot, R., Pallavicini, C., Erowid, E., \& Tagliazucchi, E. (2018). The varieties of the psychedelic experience: a preliminary study of the association between the reported subjective effects and the binding affinity profiles of substituted phenethylamines and tryptamines. Frontiers in integrative neuroscience, 12, 54. 